\title{
A broadband X-ray view of the NLSy1 1E 0754.6+392.8
}

\author{
R. Middei ${ }^{1, \star}$, F. Tombesi ${ }^{2,3,4,5}$, F. Vagnetti ${ }^{2}$, R. Serafinelli ${ }^{6}$, S. Bianchi ${ }^{1}$, \\ G. Miniutti ${ }^{7}$, A. Marinucci ${ }^{8}$, G. A. Matzeu ${ }^{9}$, P.-O. Petrucci ${ }^{10}$, F. Ursini ${ }^{11}$, and A. Zaino ${ }^{1}$
}

1 Dipartimento di Matematica e Fisica, Università degli Studi Roma Tre, via della Vasca Navale 84, I-00146 Roma, Italy

2 Dipartimento di Fisica, Università di Roma "Tor Vergata", via della Ricerca Scientifica 1, I-00133, Roma, Italy

3 INAF Astronomical Observatory of Rome, Via Frascati 33, 00078 Monteporzio Catone, Italy

4 Department of Astronomy, University of Maryland, College Park, MD 20742, USA

5 NASA/Goddard Space Flight Center, Code 662, Greenbelt, MD 20771, USA

6 INAF - Osservatorio Astronomico di Brera, Via Brera 28, 20121, Milano, Italy

7 Centro de Astrobiología (CSIC-INTA), Dep. de Astrofísica; LAEFF, Villanueva de la Cañada, Madrid, Spain

8 ASI - Unità di Ricerca Scientifica, Via del Politecnico snc, 00133 Rome, Italy

9 European Space Agency (ESA), European Space Astronomy Centre (ESAC), E-28691 Villanueva de la Cañada, Madrid, Spain

10 Univ. Grenoble Alpes, CNRS, IPAG, F-38000 Grenoble, France

11 INAF/IASF Bologna via Gobetti 101, 40129 Bologna, Italy

January 14,2020

\section{ABSTRACT}

\begin{abstract}
Context. The soft X-ray band of many active galactic nuclei (AGN) is affected by obscuration due to partially ionised matter crossing our line of sight. In this context, two past XMM-Newton observations (6 months apart) and a simultaneous NuSTAR-Swift ( $~ 8$ years later) exposure of the Narrow Line Seyfert 1 galaxy 1E 0754.6+392.8 revealed an intense and variable WA and hints of additional absorbers in the Fe K $\alpha$ band.

Aims. We aim at providing the first X-ray characterisation of this AGN discussing its broadband (0.3-79 keV) spectrum and temporal properties.

Methods. We conduct a temporal and spectroscopic analysis on two 10 ks (net exposure) XMM-Newton snapshots performed in April and October 2006. We also study the high energy behaviour of 1E $0754.6+392.8$ modelling its broadband spectrum using simultaneous Swift-NuSTAR data. Both phenomenological and physically motivated models are tested.

Results. We find the presence of flux variability ( $150 \%$ and $30 \%$ for $0.3-2$ and $2-10 \mathrm{keV}$ bands, respectively) and spectral changes at months timescales $(\Delta \Gamma \sim 0.4)$. A reflection component that is consistent with being constant over years and arising from relatively cold material far from the central super massive black hole is detected. The main spectral feature shaping the 1E $0754.6+392.8$ spectrum is a warm absorber. Such a component is persistent over the years and variability of its ionisation and column density is observed down on months in the ranges $3 \times 10^{22} \mathrm{~cm}^{-2} \lesssim \mathrm{N}_{\mathrm{H}} \lesssim 7.2 \times 10^{22} \mathrm{~cm}^{-2}$ and $1.5 \lesssim \log \left(\xi / \mathrm{erg} \mathrm{s}^{-1} \mathrm{~cm}\right) \lesssim 2.1$. Despite the short exposures, we find possible evidence of two additional high-ionisation and high-velocity outflow components in absorption.

Conclusions. Our analysis suggests the existence of a complex system of absorbers in 1E 0754.6+392.8. Longer exposures are mandatory in order to characterise, on more solid grounds, the absorbers in this AGN.
\end{abstract}

Key words. galaxies:active - galaxies:Seyfert - quasars:general - X-rays:galaxies

\section{Introduction}

Active galactic nuclei (AGNs) lie in the central region of galaxies, and their emission is observed from gamma rays down to radio frequencies. Most of the released energy is emitted in the optical-UV band due to accretion of gas from a disc surrounding a supermassive black hole $(\mathrm{SMBH}$, Event Horizon Telescope Collaboration et al. 2019). Moreover, most AGNs are luminous in the X-ray band, and this energetic emission can be explained in terms of an inverse Compton mechanism involving seed disc photons and a distribution of thermal electrons overlying the disc, the so-called hot corona (e.g. Haardt \& Maraschi 1991, 1993).

^ riccardo.middei@uniroma3.it
In X-rays, AGNs display a power law-like spectrum (e.g. Guainazzi et al. 1999, Bianchi et al. 2009), which depends on the physical conditions of the coronal plasma (i.e. electron temperature and optical depth). At hard X-ray energies, an exponential cut-off is often observed (e.g. Fabian et al. 2015, 2017, Tortosa et al. 2018), and it is interpreted as a further signature of the nuclear Comptonisation $(\overline{\mathrm{Ry}}-$ bicki \& Lightman 1979). The coronal emission can further interact with the SMBH surroundings, and the emerging spectra can be modified by absorption and reflection. The reprocessing of the primary emission gives rise to additional spectral features such as a Compton hump at about $~ 30$ keV (e.g. Matt et al. 1991; George \& Fabian 1991) and a fluorescence $\mathrm{Fe} \mathrm{K} \alpha$ emission line.

A detailed analysis of absorption profiles in X-ray spec- 
tra can provide additional information about the surroundings of the central engine. About $50 \%$ of AGNs (Reynolds 1997) display soft X-ray absorption features due to ionised gas along the line of sight which are indicative of a warm absorber (WA, Blustin et al. 2005). Such phenomenon, first reported in Halpern (1984), consists in a spectral dip, indicating distant outflowing material covering the inner Xray emission at $\sim 1 \mathrm{keV}$. High resolution spectroscopy provides the best way to discern and characterise the outflowing components (e.g. Longinotti et al. 2010, Behar et al. 2017; Laha et al. 2014, 2016; Mao et al. 2019), though low resolution studies still provide insights and can be applied on a larger number of sources (e.g. Piconcelli et al. 2005; Tombesi et al. 2010; Gofford et al. 2013 Cappi et al. 2016). The ionisation parameter of the WAs is typically in the range $\log \left(\xi /\right.$ erg s$\left.^{-1} \mathrm{~cm}\right) \simeq 0-3$ and the equivalent hydrogen column density is between $\mathrm{N}_{\mathrm{H}} \simeq 10^{20}-10^{22} \mathrm{~cm}^{-2}$. The relative absorption lines and edges are often blue-shifted, indicating that the gas is outflowing with velocities from $\mathrm{v}_{\text {out }} \simeq 100$ $\mathrm{km} \mathrm{s}^{-1}$ up to $\mathrm{v}_{\text {out }} \simeq 1000 \mathrm{~km} \mathrm{~s}^{-1}$.

Highly blueshifted Fe K absorption lines indicative of ultra fast outflows (UFOs) with velocities higher than 10000 $\mathrm{km} \mathrm{s}^{-1}$ have been reported in the X-ray spectra of several AGNs (e.g. Tombesi et al. 2010, Gofford et al. 2013). The ionisation of this outflowing plasma can be very high, in the range $\log \left(\xi / \mathrm{erg} \mathrm{s}^{-1} \mathrm{~cm}\right) \simeq 3-6$ and the column density is also large, up to values of $N_{H} \simeq 10^{24} \mathrm{~cm}^{-2}$ (e.g. Tombesi et al. 2011). Recent studies are reporting on the presence of multistructured disc winds. Reeves et al. (2018) found an additional component of the fast wind in PDS 456 while caught in the a low-flux state with XMM-Newton and NuSTAR, while the case of MCG-03-58-007 is discussed in Braito et al. (2018) and Matzeu et al. (2019). Moreover, a positive correlation between the outflow velocity of the UFOs and the X-ray luminosity (e.g. Matzeu et al. 2017; Pinto et al. 2018) has been observed, and this is expected in a radiatively driven wind scenario. These disc winds are observed at sub-parsec scales from the central SMBH and seem to be powerful enough to affect the host galaxy environment (e.g. Tombesi et al. 2012). Indeed, the recent detection of UFOs in some ultra-luminous infrared galaxies (ULIRGs) showed that they are likely responsible for driving the observed massive, large-scale interstellar matter (ISM) outflows (e.g. Tombesi et al. 2015, 2017, Feruglio et al. 2015, Fiore et al. 2017; Veilleux et al. 2017), and that they may quench star formation as expected from AGNs feedback models (e.g. Zubovas \& King 2012, Faucher-Giguère \& Quataert 2012).

In this paper we focus on the X-ray analysis of $1 \mathrm{E}$ $0754.6+392.8$. This object is one of the two brightest AGNs in the NuSTAR serendipitous source catalogue (Lansbury et al. 2017), the other being HE 0436-4717 (Middei et al. 2018b). 1E 0754.6+392.8 is classified by Berton et al. (2015) as a local $(z=0.096)$ radio quiet Narrow Line Seyfert 1 galaxy (NLS1, see also Enya et al. 2002). For the mass of the central black hole, we adopt the single-epoch estimate by Berton et al. (2015), $\log \mathrm{M}_{\mathrm{BH}} / \mathrm{M}_{\odot}=8.15$, consistent with the reverberation-based value $\log \mathrm{M}_{\mathrm{BH}} / M_{\odot}=8.0$ reported by Sergeev et al. (2007). The bolometric luminosity and Eddington ratio are estimated as $\log \left(\mathrm{L}_{\text {bol }} / \mathrm{erg} \mathrm{s}^{-1}\right)=45.4$ and $\log \left(\mathrm{L}_{\mathrm{bol}} / \mathrm{L}_{\mathrm{Edd}}\right)=-0.85$ (Berton et al. 2015$)$. Although several works discuss the optical properties of $1 \mathrm{E} 0754.6+392.8$, this source is poorly studied in the X-rays. The Einstein observatory (Giacconi et al. 1979) observed this
Table 1: The ID, the telescope, the net exposure and the start data of the observations here analysed are reported.

\begin{tabular}{ccccc}
\hline Satellite & Instrument & Obs. ID & $\begin{array}{c}\text { Net exp. } \\
(\mathrm{ks})\end{array}$ & $\begin{array}{c}\text { Start-date } \\
\text { yyyy-mm-dd }\end{array}$ \\
\hline XMM-Newton & $P N$ & 0305990101 & 13.5 & $2006-04-18$ \\
\hline XMM-Newton & $P N$ & 0406740101 & 14.7 & $2006-10-22$ \\
\hline NuSTAR & $F P M A / B$ & 60001131002 & 45.1 & $2014-09-12$ \\
\hline Swift & $X R T$ & 00080595001 & 2.7 & $2014-09-12$
\end{tabular}

AGN and Gioia et al. (1990) reported its flux to be $\mathrm{F}_{0.3-3.5 \mathrm{keV}}=1.8 \times 10^{-12}$ ergs $\mathrm{cm}^{-2} \mathrm{~s}^{-1}$. Throughout the paper, the standard cosmology $\Lambda C D M$ with $\mathrm{H}_{0}=70 \mathrm{~km} \mathrm{~s}^{-1}$ $\mathrm{Mpc}^{-1}, \Omega_{m}=0.27, \Omega_{\lambda}=0.73$, is adopted.

\section{Data reduction}

This work takes advantage of two archival XMM-Newton observations and one Swift-NuSTAR simultaneous observation (see the log in Tab. 1). The XMM-Newton (Jansen et al. 2001) observatory pointed twice $1 \mathrm{E} 0754.6+392.8$ on April 182006 and October 22 2006. NuSTAR (Harrison et al. 2013) observed the source serendipitously on September 122014 (Lansbury et al. 2017) simultaneously with Swift.

$X M M-N e w t o n$ data were processed using the XMMNewton Science Analysis System (SAS, Version 18.0.0). To select the extraction radius of each observation and to screen for high background time intervals we used an iterative process that maximises the Signal-to-Noise $(\mathrm{S} / \mathrm{N})$ ratio in the 3-9 $\mathrm{keV}$ band (details in Piconcelli et al. 2004). We therefore use a 19 arcsec radius for extracting the source in Obs. 1, while a 40 arcsec of circular region was found to maximise the $\mathrm{S} / \mathrm{N}$ ratio for Obs. 2. We extract the background of both the observations from a circular region of 50 arcsec radius located on a blank area of the detector close to the source. We binned all the spectra to have at least 25 counts for each bin, and not to oversample the instrumental energy resolution by a factor larger than 3. Data from the MOS detectors have a much lower statistics even when the spectra are co-added. Being our analysis mainly focused on the Fe K energy band, we decided to use only $P N$ due to the lower statistics of the co-added MOS spectrum.

The NuSTAR observation was reduced in accordance with the standard procedure described in Perri et al., 2013, and using HEASOFT (v. 6.25), NuSTARDAS (v 1.8.0) and the ' $\mathrm{x} 20180710$ ' version of the calibration database. Spectra were extracted for both the hard X-ray detectors $F P M A / B$ on the $N u S T A R$ focal plane. A circular extraction region with 40 arcsec radius was used for the source, while the background was obtained adopting a region of the same size in a blank area of the same chip. The $N u S T A R$ spectra were binned not to over-sample the instrumental resolution by a factor larger than of 2.5 and to have a $\mathrm{S} / \mathrm{N}$ greater than 3 in each spectral channel.

Finally, we used online facilities provided by the ASI Space Science Data Center (SSDC) for processing and reducing the Swift/XRT data (multimission archive, http:// www.ssdc.asi.it/mma.html). The spectrum was extracted from a circular region with a radius of 20 arcsec centered on the source while the background was sampled from an annular region extending between 40 arcsec and 80 arcsec around the source. For spectral fitting we use the source 


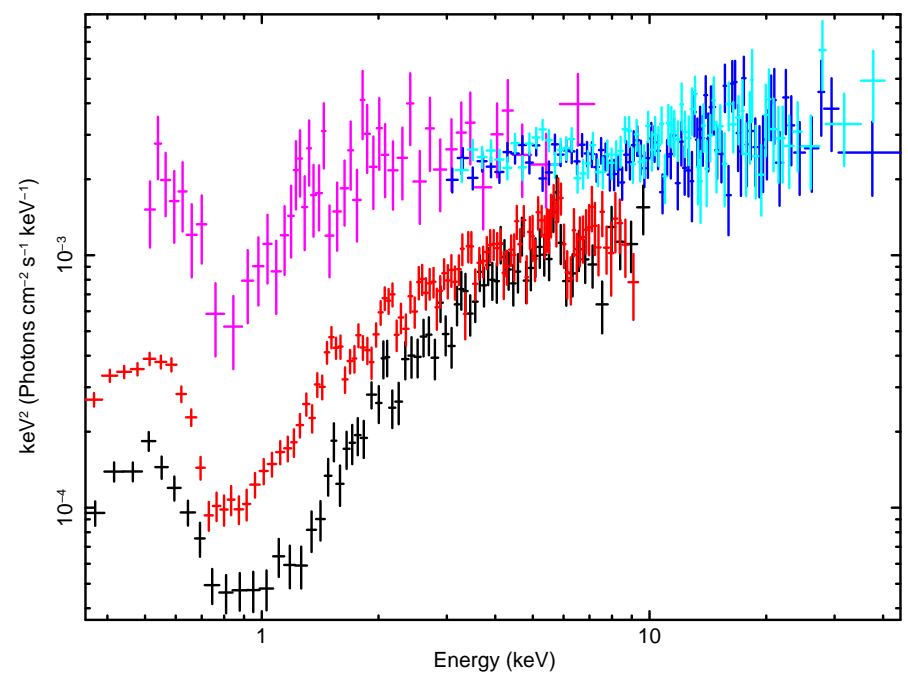

Fig. 1: The unfolded spectra of $1 \mathrm{E} 0754.6+392.8$ as observed by XMM-Newton (black for Obs. 1 and red for Obs. 2), Swift (magenta) and NuSTAR (blue and cyan). Absorption in the soft $\mathrm{X}$-rays is clearly observed and it is persistent over the years. This colour code is used throughout the paper. Finally, the underlying model consists in a power-law with $\Gamma=2$ and unitary normalisation.

spectrum binned to a minimum of 20 counts per bin.

The spectra of the whole dataset, unfolded using a $\Gamma=2$ power law model with common normalisation, are shown in Fig. 1.

\section{Spectral and timing analysis}

We consider the EPIC-pn data in the $\mathrm{E}=0.3-10 \mathrm{keV}$ energy band and the NuSTAR-Swift/XRT simultaneous observation in the $0.5-79 \mathrm{keV}$ band. We point out that $X M M$ Newton/NuSTAR exposures are about 8 years apart, thus their corresponding spectra are not simultaneously fitted. A Galactic column density of $\mathrm{N}_{\mathrm{H}}=5.6 \times 10^{20} \mathrm{~cm}^{-2}$ (HI4PI Collaboration et al. 2016) is always considered when fitting the spectra. Finally, in the text errors are quoted at $90 \%$ confidence level and errors in the plots account for $68 \%$ uncertainties.

\subsection{Swift/XRT-NuSTAR: The 0.5-79 keV band}

We investigated the temporal properties of the $N u S T A R$ observation first. The $3-10 \mathrm{keV}$ light curve reveals the presence of intra-observation variability (up to a factor of $\sim 2$ ) while a more constant behaviour characterises the 10-30 keV band, see Fig. 2. No significant spectral variability is observed, thus we considered the source spectrum integrated over the whole observation length.

To model the primary continuum, we simultaneously fit the Swift/NuSTAR data with a power law in the $3-10 \mathrm{keV}$ band. Moreover, a cross-normalisation constant is included as a free parameter to account for the different instruments involved. This crude model leads to a good fit characterised by $\chi^{2}=71$ for 66 d.o.f. and a corresponding $\Gamma=1.95 \pm 0.04$ and normalisation $\mathrm{N}_{\mathrm{po}}=(2.5 \pm 0.2) \times 10^{-3}$ photons $\mathrm{kev}^{-1} \mathrm{~cm}^{-2}$ $\mathrm{s}^{-1}$. The cross-calibration constants for Swift and NuSTAR are found to be consistent within $\sim 10 \%$, while the two $N u S$ $T A R$ modules agree each other within a $3 \%$.

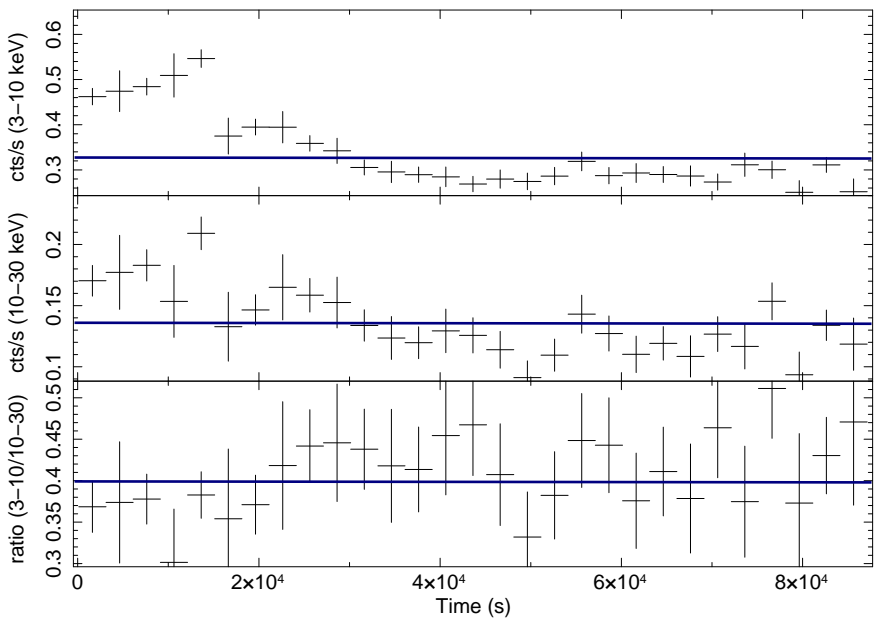

Fig. 2: The background subtracted NuSTAR light curves in the 3-10 and 10-30 keV bands are showed. In the bottom panel the ratio between the two bands is displayed. The adopted time bin is $3000 \mathrm{~s}$. The blue lines account for the average counts number in the two bands and for their ratio.

When considering the $0.5-79 \mathrm{keV}$ data, the tested model turns out to be unacceptable in terms of statistics $\left(\chi^{2}=247\right.$ for 176 d.o.f. ), mainly due to absorption affecting the soft $\mathrm{X}$-rays, see Fig. 3. To model this absorption, we included a detailed grid computed with the photoionisation code $X S$ TAR (Kallman \& Bautista 2001). This table takes into account absorption lines and edges for all the metals characterised by an atomic number $Z \leq 30$. The $X S T A R$ table is calculated assuming a typical $\Gamma=2$ for describing the spectral energy distribution in the $0.1-10^{6} \mathrm{eV}$ band, a high energy cut-off at $\mathrm{E}_{\mathrm{c}}=100 \mathrm{keV}$, and a covering factor of 1 . Elements abundance is set to the Solar one (Asplund et al. 2009 , and a turbulence velocity $\mathrm{v}_{\mathrm{t}} \simeq 200 \mathrm{~km} \mathrm{~s}^{-1}$ is considered, based on the typical values of turbulent velocity for warm absorbers (Laha et al. 2014). By letting the ionisation parameter and the column density free to vary, keeping the redshift fixed, the fit is improved by a $\Delta \chi^{2}=55$ for 2 d.o.f. less. The photon index of this new model is consistent within the errors with what previously obtained. The best-fit values of the WA are $\log \left(\xi / \mathrm{erg} \mathrm{s}^{-1} \mathrm{~cm}\right)=2.1 \pm 0.2$ and $\mathrm{N}_{\mathrm{H}}=(3 \pm 1) \times 10^{22} \mathrm{~cm}^{-2}$.

We further tested the current dataset adopting xillver (García et al. 2014; Dauser et al. 2016), a self-consistent model reproducing the continuum and ionised reflection of AGNs. In the fit, the photon index, the high energy cutoff, the reflection fraction and the normalisation are left free to vary. The iron abundance $A_{F e}$ is fixed to the Solar value, while the ionisation parameter $\xi$ is set to the lowest value allowed by the model, close to neutrality. These steps lead to the best-fit $\left(\chi^{2}=174\right.$ for 172 d.o.f.) showed in Fig. 4. The photon index and the reflection parameter are $\Gamma=2.07 \pm 0.05$ and $R=0.5 \pm 0.2$, respectively. A lower limit of $\mathrm{E}_{\mathrm{c}}>170 \mathrm{keV}$ is found for the high energy cutoff while the normalisation is $\mathrm{N}_{\mathrm{xill}}=(5.0 \pm 0.5) \times 10^{-5}$ photons $\mathrm{kev}^{-1} \mathrm{~cm}^{-2} \mathrm{~s}^{-1}$. Finally, we find that the WA parameters are still consistent within the errors with the previous fit. 


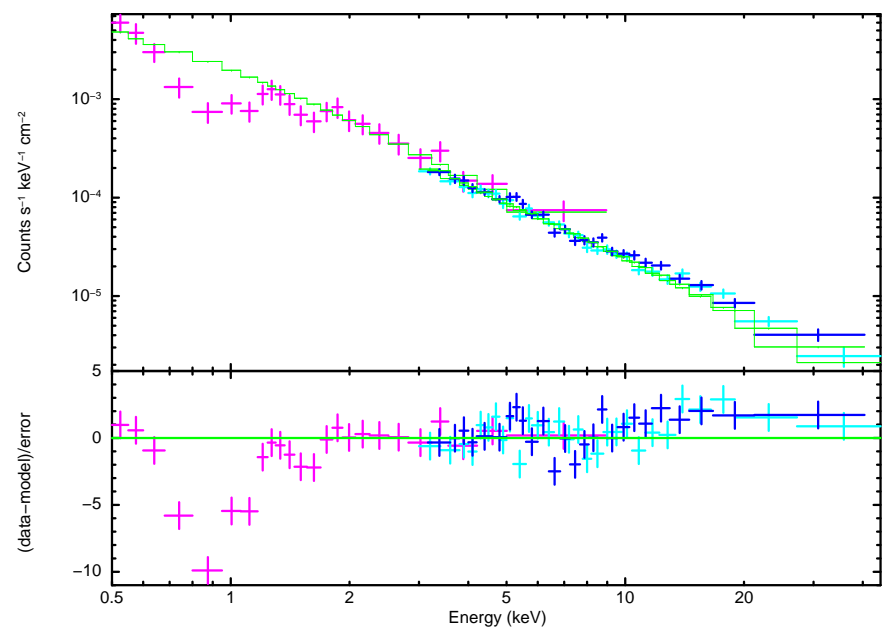

Fig. 3: The simultaneous Swift-NuSTAR observation of $1 \mathrm{E}$ $0754.6+392.8$ as fitted by a simple power-law in the $3-10 \mathrm{keV}$ band. Residuals are displayed in both soft and hard X-rays.

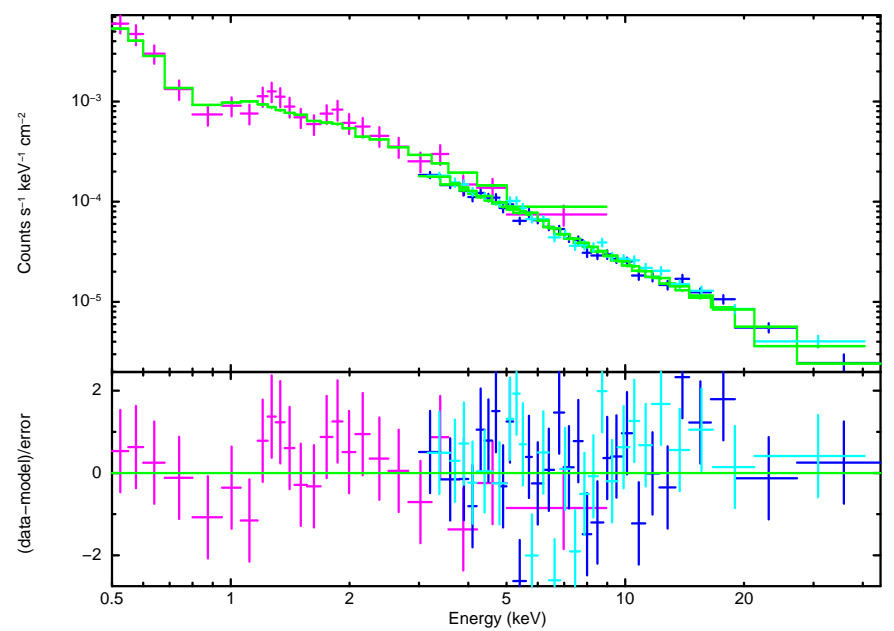

Fig. 4: The broadband best-fit $\left(\chi^{2}=174\right.$ for 172 d.o.f. $)$ to the Swift-NuSTAR observations is shown.

\subsection{XMM-Newton: The 0.3-10 keV band, analysis using a phenomenological model}

Visual inspection of the XMM-Newton light curves ( see Fig. 5) shows no evidence of flux and spectral variability, hence we used the averaged spectra to improve spectral statistics. On the other hand, the comparison of the hardness ratios between the XMM-Newton Obs. 1 and Obs. 2 suggests that the source changed its spectral shape between the two $X M M-N e w t o n$ visits.

The soft X-rays in the $X M M-N e w t o n$ observations show signs of intense absorption (see Fig. 1 and Fig. 6, panel a), therefore, we modelled the source spectra using a power-law to reproduce the underlying nuclear continuum and a $X S$ $T A R$ table to model the absorption below $\sim 2-3 \mathrm{keV}$. In the fit, the photon index the normalisation as well as the WA column density and ionisation are free to vary and untied between Obs. 1 and Obs. 2. The XSTAR table enhances the fit statistics by a $\Delta \chi^{2} /$ d.o.f $=2258 / 4$ and the resulting modelling (still unacceptable in terms of statistics, $\chi^{2}=283$ for 179 d.o.f.) is reported in Fig. 6, panel b. A prominent emission line (which its rest frame energy likely corresponds to the $\mathrm{O}$ VII transition) remains unmodelled in both pointings. We included a Gaussian line to account for it, and by fitting its energy centroid and normalisation (untied between the pointings) a corresponding $\Delta \chi^{2}=49$ for 4 parameters is found. The current model provides a fit characterised by $\chi^{2}=234$ for 175 degree of freedom.

The present model allows us to focus on the iron line energy band and, in particular, on the absorption/emission features left unmodelled in the 6-9 keV energy interval (see Fig. 6, b panel). Besides the residuals at about $\sim 6.4 \mathrm{keV}$ in emission, an absorption trough appears in the $X M M$ Newton data at the energy of $\sim 7 \mathrm{keV}$. Moreover, at higher energies, the first XMM-Newton observation shows a further absorption feature at about $\sim 8 \mathrm{keV}$. We account for these additional spectral complexities including Gaussian lines: one is used to model the $\mathrm{Fe} \mathrm{k} \alpha$ emission line and an other to reproduce the absorption feature at $\mathrm{E} \simeq 6.8 \mathrm{keV}$. During the fitting procedure, we assumed that the width of both Gaussian components is zero (unresolved by the instrument resolution) and we let the energy centroid and normalisation free to vary. We find the values for both the emission and absorption lines to be consistent between the two observations. For this reason, we fit these two Gaussian components tying the energy centroid and the normalisation between the two XMM-Newton exposures. From a statistical point of view, the inclusion of the emission line accounting for the Fe K $\alpha$ enhances the fit by $\Delta \chi^{2}=18$ for 2 d.o.f., while the absorption line at $6.8 \mathrm{keV}$ leads to a fit improvement of $\Delta \chi^{2}=11$ for 2 d.o.f.. These steps yield a global statistics of $\chi^{2}=205$ for 171 degrees of freedom. Moreover, as shown by Fig. 6 panel b, a drop of counts is observed at about $8 \mathrm{keV}$ in Obs. 1. We include an additional absorption line in our model to account for it. This line has a free energy centroid and normalisation while its intrinsic width is set fixed to $200 \mathrm{eV}$, which is comparable to the energy resolution of the EPIC-pn at these energies. A $\Delta \chi^{2}=8$ for 2 d.o.f. indicates that this component is marginally detected.

Best-fit parameters for the primary continuum and the Gaussian emission/absorption lines are reported in Table 2. Significant spectral variability is found between Obs. 1 and 2, and the power-law normalisation is found about to double in the second pointing. The observed $2-10 \mathrm{keV}$ fluxes are $(2.0 \pm 0.1) \times 10^{-12}$ and $(2.3 \pm 0.1) \times 10^{-12} \mathrm{ergs} \mathrm{cm}^{-2}$ $\mathrm{s}^{-1}$, respectively, and in the soft X-rays $(0.3-2 \mathrm{keV})$ we find $(3.0 \pm 0.2) \times 10^{-13}$ and $(7.4 \pm 0.4) \times 10^{-13} \mathrm{ergs} \mathrm{cm}^{-2} \mathrm{~s}^{-1}$. Within the errors, the intensity of emission line at energy $\sim 0.57 \mathrm{keV}$ remains constant, and the neutral $\mathrm{Fe} \mathrm{K} \alpha$ is consistent with being narrow. The Fe $\mathrm{K} \alpha$ equivalent width (EW) is also constant between the observations with an average value of $\sim 130 \mathrm{eV}$. The Gaussian line at $\sim 6.8 \mathrm{keV}$ is likely associated with blueshifted Fe XXV, while the marginally detected component at higher energy is more likely associated with a highly blueshifted Fe XXVI.

In panel c of Fig 6 and in Fig.7 we notice the presence of further features not reproduced by the current phenomenological model, especially between $1.5-2.5 \mathrm{keV}$. Some of these features in this band may be directly attributed to the detector calibration uncertainties (e.g. Si K-edge $1.84 \mathrm{keV}$ ), and at the $\mathrm{Au}$ M-edge $(\sim 2.4 \mathrm{keV})$ (see Kaastra et al. 2011 \begin{tabular}{l|l|l||}
\hline Di Gesu et al. 2015, Ursini et al. 2015, Cappi et al. 2016 \\
\hline
\end{tabular} Middei et al. 2018a, for discussions and comparisons). We notice that modelling these features with Gaussian lines or 


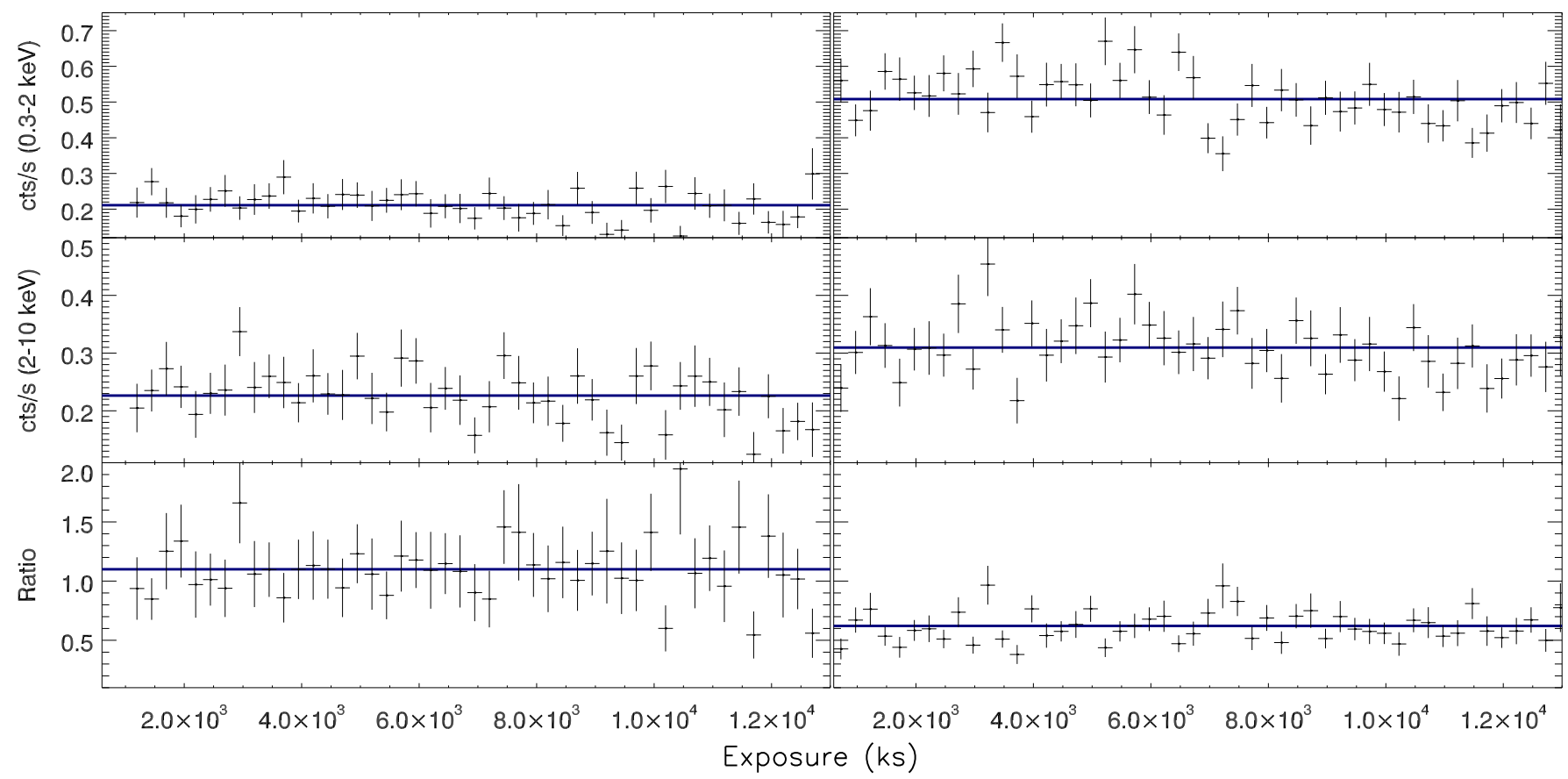

Fig. 5: The soft (0.3-2 keV) and hard (2-10 keV) background subtracted XMM-Newton light curves and their ratio are displayed. The straight blue lines account for the average rates. Left side panel refers to Obs. 1 while right hand graphs accounts for Obs. 2. The adopted bin is 250 seconds and panels share the same scale. A very weak intra-observation variability, is accompanied by remarkable flux variations between the pointings. In a similar fashion, hardness ratio are constant on ks timescales, while the source shows two different spectral states between Obs. 1 and Obs. 2.

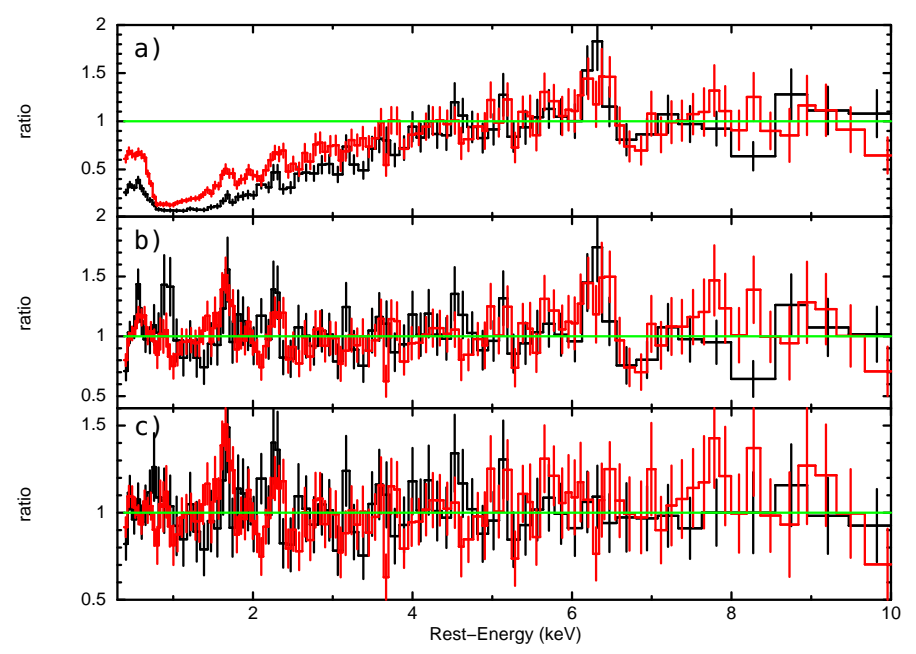

Fig. 6: Panel a: The XMM-Newton spectra with respect to a power-law only modelling in the $4-10 \mathrm{keV}$ band. Panel b: Low and high energy residuals still present after the WA was included in the modelling. Panel c: Final data-to-model ratio after the inclusion of Gaussian components. A few unmodelled features still populate the energy range between 1.5 and $2.5 \mathrm{keV}$. However some of these may be directly attributed to the detector systematics.

ignoring the spectra in the $1.5-2.5 \mathrm{keV}$ band do not affect the values reported in Table 2 .

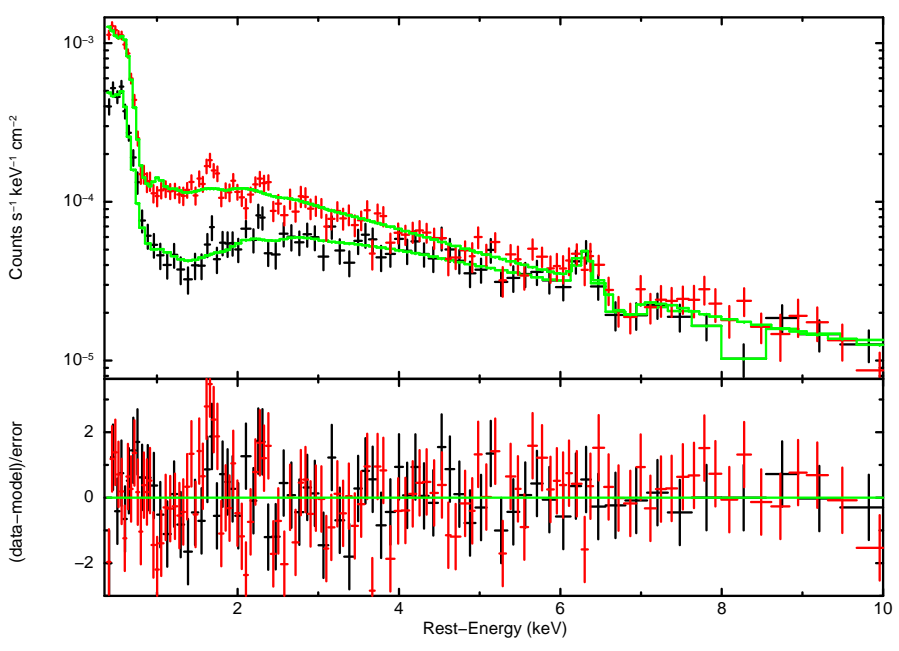

Fig. 7: The XMM-Newton EPIC-pn spectra best-fitted by the phenomenological model including an absorbed power-law and 4 Gaussian components. The corresponding residuals are shown.

\subsection{XMM-Newton: The 0.3-10 keV band, analysis using a physical model}

As a subsequent step we reanalysed the EPIC-pn spectra using a self-consistent emission model (xillver). Such a model simultaneously fits the source emission and its associated ionised reflection component. Moreover, we accounted for the absorption troughs in the $6.5-8.5 \mathrm{keV}$ energy band including two $X S T A R$ tables. One, Abs1, is used to reproduce the absorption at $\mathrm{E} \sim 6.8 \mathrm{keV}$, while, the other, Abs2 is included to model the absorption at $8 \mathrm{keV}$. The fit was 
Table 2: Parameters for the best-fit model including fully covering ionised absorption and Gaussian lines. The power law normalisation (normalised at $1 \mathrm{keV}$ ) is in units of photons $\mathrm{keV}^{-1}$ $\mathrm{cm}^{-2} \mathrm{~s}^{-1}$, while units for the column density and ionisation parameter are $10^{22} \mathrm{~cm}^{-2}$ and $\mathrm{erg} \mathrm{s}^{-1} \mathrm{~cm}$, respectively. The normalisation of emission and absorption lines are in photons $\mathrm{cm}^{-2} \mathrm{~s}^{-1}$. Finally, a $\dagger$ is used to identify the parameters kept frozen during the fit while the $\star$ specifies the component whose parameters were tied between the observations.

\begin{tabular}{|c|c|c|c|}
\hline Component & Parameter & Obs. 1 & Obs. 2 \\
\hline TBabs & $\overline{\mathrm{N}_{\mathrm{H}}^{\dagger}}$ & 0.056 & 0.056 \\
\hline \multirow[t]{2}{*}{ power-law } & $\Gamma$ & $1.66 \pm 0.04$ & $2.05 \pm 0.10$ \\
\hline & Norm $\left(\times 10^{-3}\right)$ & $0.64 \pm 0.1$ & $1.4 \pm 0.2$ \\
\hline \multirow[t]{3}{*}{ WA } & $\log \xi$ & $2.00 \pm 0.04$ & $1.75 \pm 0.15$ \\
\hline & $N_{\mathrm{H}}$ & $\begin{array}{c}7.9 \pm 0.9 \\
0.096\end{array}$ & $5.4 \pm 0.4$ \\
\hline & $\Delta \chi^{2} /$ d.o.f. & $642 / 2$ & $1616 / 2$ \\
\hline \multirow[t]{5}{*}{ zgauss (Emi) } & $\mathrm{E}(\mathrm{keV})$ & $0.56 \pm 0.02$ & $0.59 \pm 0.02$ \\
\hline & $z \dagger$ & 0.096 & - \\
\hline & Norm $\left(\times 10^{-4}\right)$ & $1.4 \pm 0.4$ & $2.6 \pm 1.0$ \\
\hline & $\mathrm{EW}(\mathrm{eV})$ & $70 \pm 30$ & $45 \pm 25$ \\
\hline & $\Delta \chi^{2} /$ d.o.f. & $18 / 2$ & $31 / 2$ \\
\hline \multirow[t]{5}{*}{ zgauss (Emi) } & $\mathrm{E}(\mathrm{keV})$ & $6.30 \pm 0.8$ & \\
\hline & $z \dagger$ & 0.096 & \\
\hline & Norm $\left(\times 10^{-6}\right)$ & $5.0 \pm 2.0$ & \\
\hline & $\mathrm{EW}(\mathrm{eV})$ & $130 \pm 90$ & $125 \pm 80$ \\
\hline & $\Delta \chi^{2} /$ d.o.f. & $18 / 2$ & \\
\hline \multirow[t]{5}{*}{ zgauss $(\mathrm{Abs}) \star$} & $\mathrm{E}(\mathrm{keV})$ & $6.80 \pm 0.07$ & \\
\hline & $z \dagger$ & 0.096 & \\
\hline & Norm $\left(\times 10^{-6}\right)$ & $-3.5 \pm 1.2$ & \\
\hline & EW (eV) & $-100 \pm 50$ & $-100 \pm 50$ \\
\hline & $\Delta \chi^{2} /$ d.o.f. & $11 / 2$ & \\
\hline \multirow[t]{6}{*}{ zgauss (Abs) } & $\mathrm{E}(\mathrm{keV})$ & $8.2 \pm 0.2$ & \\
\hline & $\sigma \dagger(\mathrm{eV})$ & 200 & - \\
\hline & $z^{\dagger}$ & 0.096 & - \\
\hline & Norm $\left(\times 10^{-6}\right)$ & $-5.4 \pm 3.1$ & - \\
\hline & $\mathrm{EW}(\mathrm{eV})$ & $-220 \pm 120$ & - \\
\hline & $\Delta \chi^{2} /$ d.o.f. & $8 / 2$ & \\
\hline
\end{tabular}

performed allowing the photon index, the reflection fraction and the normalisation to vary also between the two pointings. The high energy cut-off is kept frozen to $E_{\mathrm{c}}=100 \mathrm{keV}$, while the ionisation parameter is free to vary but tied between Obs. 1 and Obs. 2. Concerning the ionised absorbers, we fit the ionisation parameter and column density in both the observations. The table accounting for the drop at 6.8 $\mathrm{keV}$ was fitted tying its parameters between the two $X M M$ Newton observations, while the grid accounting for Abs2 was only included in Obs. 1. Finally, we considered the redshift of all the tables $\left(\mathrm{z}_{\mathrm{obs}}\right)$ as being a free parameter in order to constrain the possible velocity shift.

The steps just described lead to the best-fit in Fig. 9 . The fit has a statistics of $\Delta \chi^{2}=170$ for 168 d.o.f. and the corresponding best-fit values of the various parameters are reported in Table 3. As already shown by the phenomenological model, spectral variability characterises the primary continuum of $1 \mathrm{E} 0754.6+392.8$. The nuclear emission normalisation increases by a factor $\sim 1.25$ between the two $X M M-N e w t o n$ visits, while the reflection fraction $R$ is found to be $\sim 1$ and constant within the uncertain-
Table 3: Values and corresponding uncertainties for the best-fit parameters are shown. The overall fit statistics is $\Delta \chi^{2}=170$ for 168 d.o.f. . The $\Delta \chi^{2}$ and the corresponding variation of degrees of freedom are also reported. For Abs1 and Abs2 the turbulence velocities are $300 \mathrm{~km} \mathrm{~s}^{-1}$ and $10000 \mathrm{~km} \mathrm{~s}^{-1}$, respectively

\begin{tabular}{cccc}
\hline Model & Parameter & Obs. 1 & Obs. 2 \\
& & & \\
\hline \hline TBabs & $\mathrm{N}_{\mathrm{H}} \dagger$ & 0.056 & 0.056 \\
\hline xillver & $\Gamma$ & $1.66 \pm 0.04$ & $2.07 \pm 0.06$ \\
& $\log \xi \dagger$ & $1.3_{-0.2}^{+0.1}$ & \\
& $\mathrm{R}$ & $1.0 \pm 0.5$ & $1.0 \pm 0.4$ \\
& $\mathrm{Norm}\left(\times 10^{-5}\right)$ & $1.5 \pm 0.2$ & $2.0 \pm 0.1$ \\
\hline WA & $\log \xi$ & $2.00 \pm 0.05$ & $1.50 \pm 0.07$ \\
& $\mathrm{~N}_{\mathrm{H}}\left(\times 10^{22} \mathrm{~cm}^{-2}\right)$ & $7.0 \pm 1.0$ & $4.0 \pm 0.5$ \\
& $\mathrm{v}_{\text {out }} / c$ & $<0.005$ & $<0.004$ \\
& $\mathrm{v}_{\text {out }}\left(\mathrm{km} \mathrm{s}^{-1}\right)$ & $<1500$ & $<1100$ \\
& $\Delta \chi^{2} /$ d.o.f. & $482 / 3$ & $1171 / 3$ \\
\hline Abs1 $\star$ & $\log \xi$ & $3.4 \pm 0.1$ & \\
& $\mathrm{~N}_{\mathrm{H}}\left(\times 10^{23} \mathrm{~cm}^{-2}\right)$ & $2.6_{-1.8}^{+2.2}$ & \\
& $\mathrm{v}_{\text {out }} / c$ & $0.017 \pm 0.04$ & \\
& $\mathrm{v}_{\text {out }}\left(\mathrm{km} \mathrm{s} \mathrm{s}^{-1}\right)$ & $5300 \pm 900$ & \\
& $\Delta \chi^{2} /$ d.o.f. & $23 / 3$ & \\
\hline Abs2 & $\log \xi$ & $3.4 \pm 0.3$ & - \\
& $\mathrm{N}_{\mathrm{H}}\left(\times 10^{23} \mathrm{~cm}^{-2}\right)$ & $1.3_{-0.8}^{+1.0}$ & - \\
& $\mathrm{v}_{\text {out }} / c$ & $0.23 \pm 0.02$ & - \\
& $\mathrm{v}_{\text {out }}\left(\mathrm{km} \mathrm{s} \mathrm{s}^{-1}\right)$ & $74000 \pm 10000$ & \\
& $\Delta \chi^{2} /$ d.o.f. & $14 / 3$ & -
\end{tabular}

ties. The WA component varies both in column density and ionisation in the range $3 \times 10^{22} \mathrm{~cm}^{-2} \lesssim \mathrm{N}_{\mathrm{H}} \lesssim 7 \times 10^{22} \mathrm{~cm}^{-2}$ and $1.5 \lesssim \log \left(\xi / \mathrm{erg} \mathrm{s}^{-1} \mathrm{~cm}\right) \lesssim 2.1$, respectively. These values, though slightly smaller, are consistent within the errors with those of the phenomenological model. Higher ionisations states and column densities characterise both Abs1 and Abs2. The physical parameters of these two components are marginally constrained by the current dataset and in Fig. 8 we show the confidence regions for the $\log \xi$ vs $N_{H}$ parameters. Using the redshift best-fit values, we can only found an upper limit of $\mathrm{v}_{\text {out }} \leq 1500 \mathrm{~km} \mathrm{~s}^{-1}$ for the WA. Abs1 is consistent with being in outflow with a velocity $\mathrm{v}_{\text {out }}$ in the range $4400-6200 \mathrm{~km} \mathrm{~s}^{-1}$, while a $\mathrm{v}_{\text {out }}=(0.23 \pm 0.02) c(c$ being the speed of light) is estimated for the possible Abs2 component. These physical quantities for various absorbers in $1 \mathrm{E} 0754.6+392.8$ are compatible with what is found by other authors (e.g. Tombesi et al. 2010, 2013) with the exception of the WA column density that appears to be larger than typical values, but it could be related to a more equatorial inclination of this AGN (see also Krolik \& Kriss 2001, Behar et al. 2017).

\subsection{Comparison between XMM-Newton and Swift/NuSTAR data}

The current dataset covers a $\sim 8$ years long time interval, thus it is suitable for variability studies. As showed in Fig. 1 , the spectra of $1 \mathrm{E} 0754.6+392.8$ varied both in shape and amplitude. The observed flux in the $0.5-2 \mathrm{keV}$ band exhibits a change between the XMM-Newton exposures and in the subsequent Swift-NuSTAR pointing in which it increased by a factor larger than 10 . On the other hand, the observed $3-10 \mathrm{keV}$ flux is fairly consistent with $\mathrm{F}_{3-10 \mathrm{keV}} \sim 2 \times 10^{-12}$ 


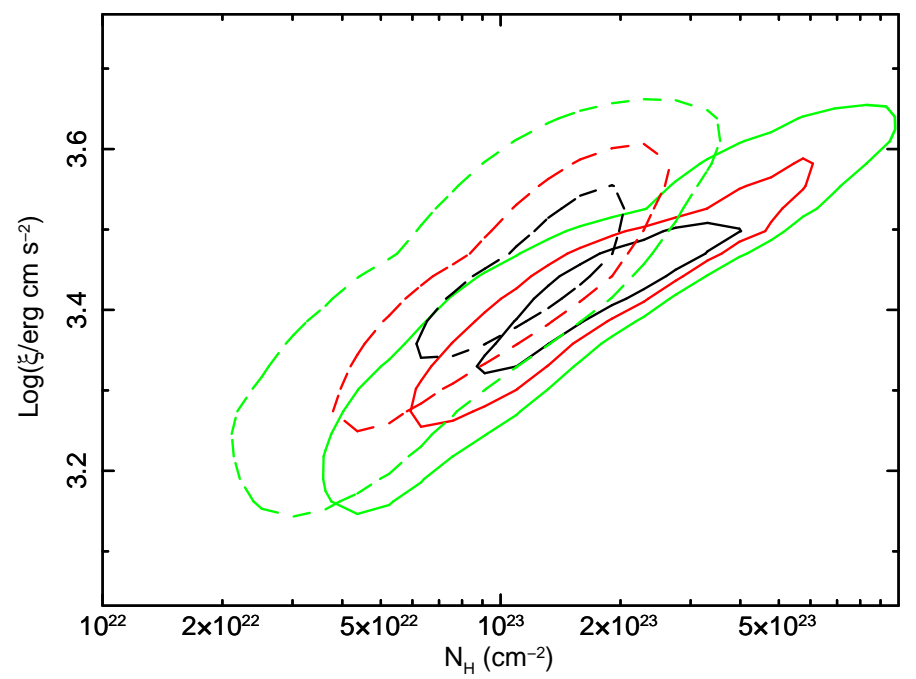

Fig. 8: The contours at 99\% (green), 90\%(red) and 68\% (black) confidence level computed for Abs1 (solid lines) and Abs2 (dashed lines).

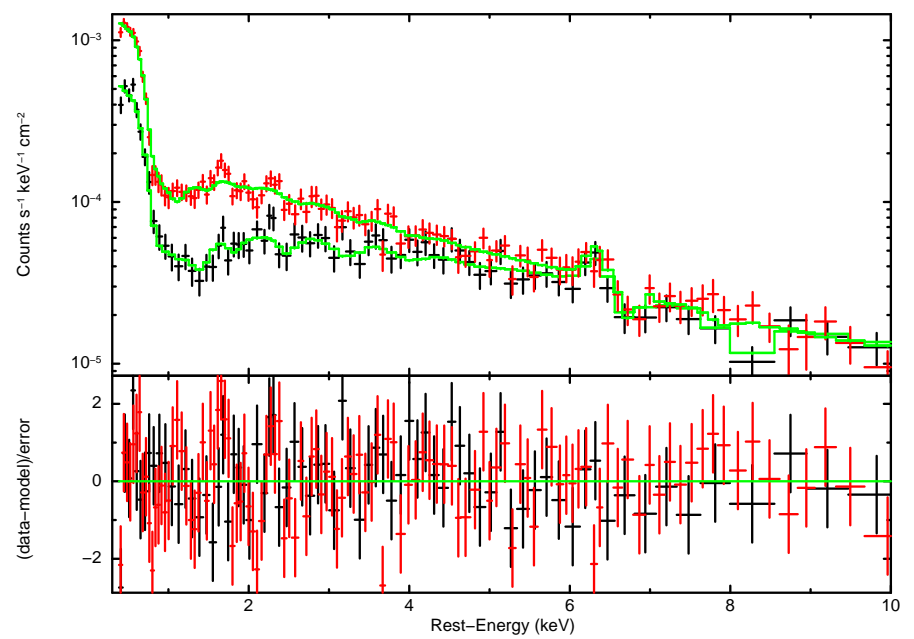

Fig. 9: The best-fit model to the two $X M M$-Newton observations of the model including three different XSTAR tables and xillver.

erg $\mathrm{cm}^{-2} \mathrm{~s}^{-1}$ in Obs. 1 and Obs. 2, while the source doubled the flux in the same band during the Swift-NuSTAR observation. It is worth noting that the reflection fraction $R$ is higher when the $3-10 \mathrm{keV}$ flux is lower, suggesting a constant reflected emission. Intense absorption in the soft $\mathrm{X}$-rays is the major component that shapes the source spectrum.

The WA varies in ionisation and column density. For the physical parameters of this component and using the self-consistent models in Sect. 3.1 and 3.3 we computed the confidence regions showed in Fig. 10. These contour plots have been calculated by assuming the redshift of the $X S$ $T A R$ tables fixed at its best-fit value. In Fig. 10, the low statistics of the XRT data and the FPMA $\& B$ bandpass explain the poor constraints on the WA in the 2014 observation.

Moreover, we tested Swift-NuSTAR data for the presence of absorption lines. We started with the phenomenological model presented in Sect. 3.1 to which we have added Gaussian components in absorption: a narrow line with an energy centroid of $6.8 \mathrm{keV}$; a $200 \mathrm{eV}$ width absorp-

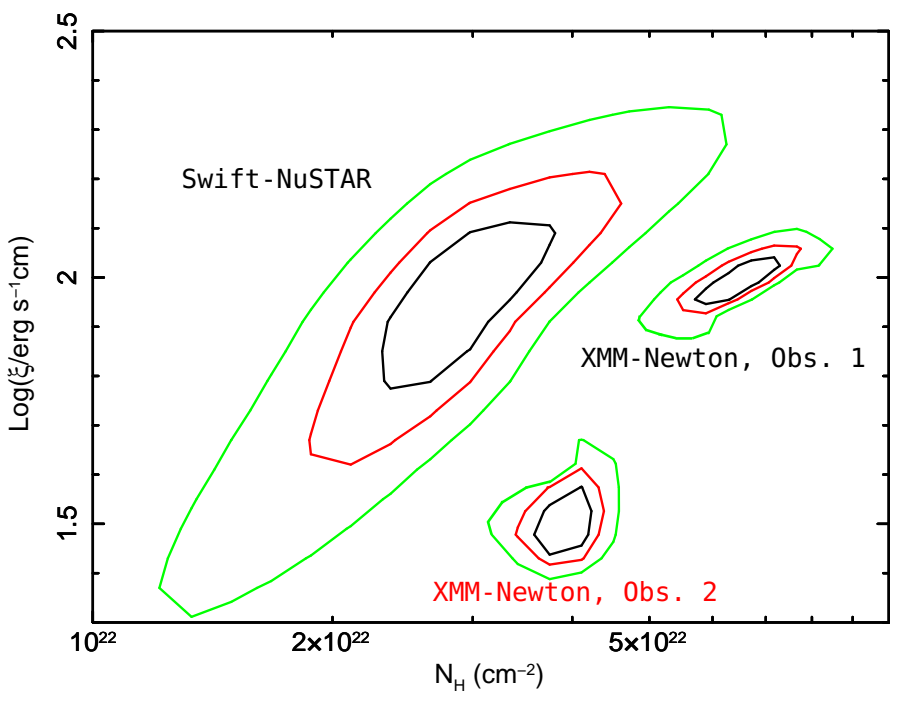

Fig. 10: The $68 \%$ (black), $90 \%$ (red) and $99 \%$ (green) confidence level contours are plotted for the column density and ionisation state of the WA component in $1 \mathrm{E} 0754.6+392.8$, respectively. Such a component is clearly monthly variable and, though with higher uncertainties due to the poorer statistics of the $X R T$ observation, over the years.

tion line centered at $8.2 \mathrm{keV}$. The fit to the data does not require a Gaussian component at $6.8 \mathrm{keV}$. On the other hand, the other line provides a weak improvement of the fit $\left(\Delta \chi^{2} /\right.$ d.o.f. $\left.=6 / 2\right)$ and the absorption line is characterised by $\mathrm{E}=8.9 \pm 0.3 \mathrm{keV}, \mathrm{N}=(6.2 \pm 4.0) \times 10^{-6}$ photons $\mathrm{cm}^{-2}$ $\mathrm{s}^{-1} \mathrm{EW}=-100 \pm 60 \mathrm{eV}$. However, the statistics of the available Swift-NuSTAR exposure is not suitable for adequately searching for faint absorption features.

\section{Statistical significance of the absorption features}

In order to assess the statistical significance of the two absorption features at $\sim 6.8$ and $\sim 8 \mathrm{keV}$, we performed Monte Carlo simulations. These simulations are particularly suitable for quantifying the correct significance of any absorption/emission component detected with a blind search over a certain energy interval (e.g. Porquet et al. 2004, Miniutti \& Fabian 2006, Markowitz et al. 2006, Tombesi et al. 2010 Gofford et al. 2013; Tombesi \& Cappi 2014; Marinucci et al. 2018: Smith et al. 2019). Therefore, we used the fakeit command in XSPEC to generate a set of 1000 synthetic spectra for each of the two exposures. To simulate these fake spectra, we used the background and response files of the real data and the same exposure time of the observations. The underlying model considered for the simulations is the one presented in Sect. 3.2 but without the emission and absorption lines. Finally, the simulated data were binned in the same way as those observed. After that, we add a new narrow (or with a width of $200 \mathrm{eV}$, for the candidate UFO) Gaussian line whose normalisation was initially set to zero and free to vary in the range between -1 and +1 . The energy centroid was free to vary between 6.5 and $9 \mathrm{keV}$ for both the features, in order to sample the searched energy interval. We used the steppar command in XSPEC to map the $\Delta \chi^{2}$ using $100 \mathrm{eV}$ steps, and the resulting variation where recorded.

Defining $\mathrm{N}$ as the number of simulations in which a chance improvement of the $\chi^{2}$ is found to be equal or larger 
than the one on the real data and $\mathrm{S}$ being the total number of simulated spectra, we estimated the Monte Carlo statistical significance of the detections to be 1-N/S. Following this definition, we obtained $\mathrm{N}=16$ with $\mathrm{S}=1000$ for the absorption line at $6.8 \mathrm{keV}$. Hence the significance of this feature in accordance with the simulations is $98.4 \%$, this corresponding to a $2.4 \sigma$ detection. For the candidate UFO at E 8 $\mathrm{keV}$, we find $\mathrm{N}=39$ which corresponds to a significance of $96.1 \%$ i.e. $2.06 \sigma$. We note that the Monte Carlo statistical significance of these features is higher than the threshold of $95 \%$ typically used in extensive searches of Fe $\mathrm{K}$ features (e.g. Tombesi et al. 2010, 2014, Gofford et al. 2013).

\section{Conclusions and summary}

We reported on the first X-ray broadband (0.3-79 $\mathrm{keV})$ spectroscopic analysis of the NLSy1 galaxy $1 \mathrm{E}$ 0754.6+392.8 based on two 2006 XMM-Newton observations (taken 6 months apart) and on a NuSTAR-Swift simultaneous snapshot performed in 2014. The spectra of $1 \mathrm{E}$ $0754.6+392.8$ are well described by a variable power-law spectrum with a photon index between 1.65 and 2.07. This spectral variability is observed from months to years, while down to hours the source exhibits a constant behaviour as suggested by the hardness ratios in Fig. 2 and Fig. 5). Long term flux variations mainly affect the soft X-rays (0.3$2 \mathrm{keV}$ ), whose flux doubles in six months and increases more than a factor 10 in 8 years. On the other hand, the continuum emission at higher energies is less affected by variations on monthly timescales, but, over the years, the observed 2 $10 \mathrm{keV}$ flux increased by a factor $\sim 2.5$.

Two significant emission lines are detected at $\sim 6.4 \mathrm{keV}$ and $\sim 0.57 \mathrm{keV}$, respectively. The former is interpreted as fluorescent emission of K-shell iron in a low-ionisation state. Its width is unresolved in both the observations (upper limits $\sigma_{\mathrm{Fe} \alpha}<0.19 \mathrm{keV}$ ), and this may rule out an origin in the inner parts of the accretion disc. Furthermore, such emission feature has a constant equivalent width and normalisation. The reflected flux is consistent with being constant over the years and it is likely originating in distant material. The other feature in emission observed at about $\sim 0.57$ $\mathrm{keV}$ may result from $\mathrm{He}$-like oxygen triplet emission arising from the same low-ionisation state plasma responsible for the iron $\mathrm{K}$ emission line. However, such line may also be produced in a much farther region from the SMBH (e.g. the Narrow Line Region) and it is more easily detected due to the presence of the WA itself (Piconcelli et al. 2004 ).

The main spectral feature in the spectrum is an intense absorption affecting the soft X-ray band. In particular, data show a variable WA that is persistent over the years. This component is consistent with being at the same redshift of the source and at pc scales with respect to the central SMBH. The physical quantities derived for the WA are shown in Fig. 10 for the different observations. The change in column density and/or ionisation state of this component can be the result of a clumpy or filamentary inhomogeneous absorber (e.g. Gaspari \& Sądowski 2017, Serafinelli et al. 2019).

The iron line energy band shows further signatures of absorption likely due to highly ionised and high column density matter crossing our line of sight. Though, these components have only a low significance ( $\sim 98 \%$ for Fe XxV and $\sim 96 \%$ for Fe XXVI assessed using Monte-Carlo simulations). The Fe K absorber Abs1 is observed in both XMM-
Newton observations and is consistent with a Fe Xxv. Abs2, a candidate UFO, is characterised by a mildly outflowing velocity $\left(\mathrm{v}_{\text {out }}=(0.23 \pm 0.03) c\right)$ and its ionisation and column density are compatible with what often observed for UFOs (Tombesi et al. 2011; Parker et al. 2017; Reeves et al. 2018, Parker et al. 2018, Braito et al. 2018; Serafinelli et al. 2019. Matzeu et al. 2019).

The presence/absence of the Abs2 component in the analysed data is consistent with SMBH winds being variable as it has been repeatedly confirmed through ensemble studies or single object analyses. For instance, Tombesi et al. (2010) reported on the variability of such winds using a sample of Seyfert galaxies and found a detection rate of the order of $50 \pm 20 \%$ for these components (see also Tombesi et al. 2011; Gofford et al. 2013).

It is worth noticing that the various types of absorbers can be part of a single large-scale multiphase outflow seen at different distances from the SMBH (e.g. Tombesi et al. 2013). The properties of the WAs, the UFOs and the highly ionised non-UFO absorbers (like as our Abs1) have been found to show significant trends: the closer the absorber is to the central $\mathrm{BH}$, the higher the ionisation, column, outflow velocity. Within this context, the possible simultaneous presence of three different types of absorbers suggests $1 \mathrm{E} 0754.6+392.8$ to be a fantastic laboratory in which to study the relations between the different absorbing phases. In fact, though being rarely observed so far, the presence of multiple phases allows unprecedented insights on the outflows structure and physics (e.g. Serafinelli et al. 2019).

In conclusion, the current data, despite the low $\mathrm{S} / \mathrm{N}$, suggest that $1 \mathrm{E} 0754.6+392.8$, clearly hosting a variable WA, may have further absorbing phases characterised by much higher outflow velocities. Only longer exposures or the higher sensitivity of an X-ray calorimeter (e.g. XRISM and Athena) will allow us to put firmer conclusions on the putative multiphase outflows possibly present on this source and to better assess for the presence of its accretion disc wind component.

Acknowledgements. We thank the referee for her/his suggestions which improved the manuscript. RM thanks Valentina Braito and James Reeves for useful discussions and comments. RM acknowledges support from the Faculty of the European Space Astronomy Centre (ESAC), Fondazione Angelo Della Riccia for financial support and Université Grenoble Alpes and the high energy SHERPAS group for welcoming him at IPAG. FT acknowledges support by the Programma per Giovani Ricercatori - anno 2014 "Rita Levi Montalcini". Part of this work is based on archival data, software or online services provided by the Space Science Data Center - ASI. SB and AZ acknowledge financial support from ASI under grants ASI-INAF I/037/12/0 and n. 2017-14-H.O. RS acknowledges financial contribution from the agreement ASI-INAF n.2017-14-H.0. POP thanks financial support by the french CNES agency. GAM is supported by European Space Agency (ESA) Research Fellowships. This work is based on observations obtained with: the NuSTAR mission, a project led by the California Institute of Technology, managed by the Jet Propulsion Laboratory and funded by NASA; XMM-Newton, an ESA science mission with instruments and contributions directly funded by ESA Member States and the USA (NASA).

\section{References}

Asplund, M., Grevesse, N., Sauval, A. J., \& Scott, P. 2009, ARA\&A, 47, 481

Behar, E., Peretz, U., Kriss, G. A., et al. 2017, A\&A, 601, A17 Berton, M., Foschini, L., Ciroi, S., et al. 2015, A\&A, 578, A28 Bianchi, S., Guainazzi, M., Matt, G., Fonseca Bonilla, N., \& Ponti, G. 2009, A\&A, 495, 421 
Blustin, A. J., Page, M. J., Fuerst, S. V., Branduardi-Raymont, G., \& Ashton, C. E. 2005, A\&A, 431, 111

Braito, V., Reeves, J. N., Matzeu, G. A., et al. 2018, MNRAS, 479, 3592

Cappi, M., De Marco, B., Ponti, G., et al. 2016, A\&A, 592, A27
Dauser, T., García, J., Walton, D. J., et al. 2016, A\&A, 590, A76

Di Gesu, L., Costantini, E., Ebrero, J., et al. 2015, A\&A, 579, A42

Event Horizon Telescope Collaboration, Akiyama, K., Alberdi, A., et al. 2019, ApJ, 875, L5

Fabian, A. C., Lohfink, A., Belmont, R., Malzac, J., \& Coppi, P. 2017, MNRAS, 467, 2566

Fabian, A. C., Lohfink, A., Kara, E., et al. 2015, MNRAS, 451, 4375

Faucher-Giguère, C.-A. \& Quataert, E. 2012, MNRAS, 425, 605

Feruglio, C., Fiore, F., Carniani, S., et al. 2015, A\&A, 583, A99

Fiore, F., Feruglio, C., Shankar, F., et al. 2017, A\&A, 601, A143

García, J., Dauser, T., Lohfink, A., et al. 2014, ApJ, 782, 76

Gaspari, M. \& Sacdowski, A. 2017, ApJ, 837, 149

George, I. M. \& Fabian, A. C. 1991, MNRAS, 249, 352

Giacconi, R., Branduardi, G., Briel, U., et al. 1979, ApJ, 230, 540

Gioia, I. M., Maccacaro, T., Schild, R. E., et al. 1990, ApJS, 72, 567

Gofford, J., Reeves, J. N., Tombesi, F., et al. 2013, MNRAS, 430, 60

Guainazzi, M., Matt, G., Molendi, S., et al. 1999, A\&A, 341, L27

Haardt, F. \& Maraschi, L. 1991, ApJ, 380, L51

Haardt, F. \& Maraschi, L. 1993, ApJ, 413, 507

Halpern, J. P. 1984, ApJ, 281, 90

Harrison, F. A., Craig, W. W., Christensen, F. E., et al. 2013, ApJ, 770,103

HI4PI Collaboration, Ben Bekhti, N., Flöer, L., et al. 2016, A\&A, 594, A116

Jansen, F., Lumb, D., Altieri, B., et al. 2001, A\&A, 365, L1

Kaastra, J. S., Petrucci, P.-O., Cappi, M., et al. 2011, A\&A, 534, A36

Kallman, T. \& Bautista, M. 2001, ApJS, 133, 221

Krolik, J. H. \& Kriss, G. A. 2001, The Astrophysical Journal, 561, 684

Laha, S., Guainazzi, M., Chakravorty, S., Dewangan, G. C., \& Kembhavi, A. K. 2016, MNRAS, 457, 3896

Laha, S., Guainazzi, M., Dewangan, G. C., Chakravorty, S., \& Kembhavi, A. K. 2014, MNRAS, 441, 2613

Lansbury, G. B., Alexander, D. M. Aird, J, et al. 2017, ApJ, 846, 20

Longinotti, A. L., Costantini, E., Petrucci, P. O., et al. 2010, A\&A, 510, A92

Mao, J., Mehdipour, M., Kaastra, J. S., et al. 2019, A\&A, 621, A99
Marinucci, A., Bianchi, S., Braito, V., et al. 2018, MNRAS, 478, 5638

Markowitz, A., Reeves, J. N., \& Braito, V. 2006, ApJ, 646, 783

Matt, G., Perola, G. C., \& Piro, L. 1991, A\&A, 247, 25

Matzeu, G. A., Braito, V., Reeves, J. N., et al. 2019, MNRAS, 483, 2836

Matzeu, G. A., Reeves, J. N., Braito, V., et al. 2017, MNRAS, 472 L15

Middei, R., Bianchi, S., Cappi, M., et al. 2018a, A\&A, 615, A163

Middei, R., Vagnetti, F., Tombesi, F., et al. 2018b, A\&A, 618, A167

Miniutti, G. \& Fabian, À. C. 2006, MNRAS, 366, 115

Parker, M. L., Matzeu, G. A., Guainazzi, M., et al. 2018, MNRAS, 480, 2365

Parker, M. L., Pinto, C., Fabian, A. C., et al. 2017, Nature, 543, 83

Piconcelli, E., Jimenez-Bailón, E., Guainazzi, M., et al. 2004, MNRAS, 351,161

Piconcelli, E., Jimenez-Bailón, E., Guainazzi, M., et al. 2005, A\&A, 432, 15

Pinto, C., Alston, W., Parker, M. L., et al. 2018, MNRAS, 476, 1021

Porquet, D., Reeves, J. N., Uttley, P., \& Turner, T. J. 2004, A\&A, 427, 101

Reeves, J. N., Braito, V., Nardini, E., et al. 2018, ApJ, 854, L8

Reynolds, C. S. 1997, MNRAS, 286, 513

Rybicki, G. B. \& Lightman, A. P. 1979, Radiative processes in astrophysics

Serafinelli, R., Tombesi, F., Vagnetti, F., et al. 2019, A\&A, 627, A121

Sergeev, S. G., Klimanov, S. A., Chesnok, N. G., \& Pronik, V. I. 2007 Astronomy Letters, 33, 429

Smith, R. N., Tombesi, F., Veilleux, S., Lohfink, A. M., \& Luminari, A. 2019, arXiv e-prints, arXiv:1910.14583

Tombesi, F. \& Cappi, M. 2014, MNRAS, 443, L104

Tombesi, F., Cappi, M., Reeves, J. N., \& Braito, V. 2012, MNRAS, 422, L1

Tombesi, F., Cappi, M., Reeves, J. N., et al. 2013, MNRAS, 430, 1102

Tombesi, F., Cappi, M., Reeves, J. N., et al. 2011, ApJ, 742, 44

Tombesi, F., Cappi, M., Reeves, J. N., et al. 2010, A\&A, 521, A57

Tombesi, F., Meléndez, M., Veilleux, S., et al. 2015, Nature, 519, 436

Tombesi, F., Tazaki, F., Mushotzky, R. F., et al. 2014, MNRAS, 443, 2154

Tombesi, F., Veilleux, S., Meléndez, M., et al. 2017, ApJ, 850, 151

Tortosa, A., Bianchi, S., Marinucci, A., Matt, G., \& Petrucci, P. O. 2018, A\&A, 614, A37

Ursini, F., Boissay, R., Petrucci, P.-O., et al. 2015, A\&A, 577, A38

Veilleux, S., Bolatto, A., Tombesi, F., et al. 2017, ApJ, 843, 18

Zubovas, K. \& King, A. 2012, ApJ, 745, L34 